\title{
Agreement between the Schedule for the Evaluation of Individual Quality of Life- Direct Weighting (SEIQoL-DW) interview and a paper-administered adaption
}

Marion Burckhardt ${ }^{1,2}$, Steffen Fleischer ${ }^{1}$ and Almuth Berg ${ }^{1 *}$

\begin{abstract}
Background: The Schedule for the Evaluation of Individual Quality of Life-Direct Weighting (SEIQoL-DW) is a prevalent face-to-face interview method for measuring quality of life by integrating respondent-generated dimensions. To apply this method in clinical trials, a paper-administered alternative would be of interest. Therefore, our study aimed to analyze the agreement between the SEIQoL-DW and a paper questionnaire version (SEIQoL-PF/G).
\end{abstract}

Methods: In a crossover design, both measures were completed in a random sequence. 104 patients at a heart surgery hospital in Germany were randomly assigned to receive either the SEIQoL-DW or the SEIQoL-PF/ $\mathrm{G}$ as the first measurement in the sequence. Patients were approached on their earliest stable day after surgery. The average time between both measurements was 1 day (mean 1.3; SD 0.8).

Agreement regarding the indices, ratings, and weightings of nominated life areas (cues) was explored using Bland-Altman plots with 95\% limits of agreement (LoA). Agreement of the SEIQoL indices was defined as acceptable if the LoA did not exceed a threshold of 10 scale points. Data from $n=99$ patients were included in the agreement analysis.

Results: Both measures led to similarly nominated cues. The most frequently nominated cues were "physical health" and "family".

In the Bland-Altman plot, the indices showed a mean of differences of 2 points $(95 \% \mathrm{Cl},-1$ to 6$)$. The upper LoA showed a difference of 36 points ( $95 \% \mathrm{Cl}, 30$ to 42), and the lower LoA showed a difference of -31 points $(95 \% \mathrm{Cl},-37$ to -26$)$.

Thus, the LoAs and confidence intervals exceeded the predefined threshold. The Bland-Altman plots for the cue levels and cue weights showed similar results.

The SEIQoL-PF/G version showed a tendency for equal weighting of cues, while the weighting procedure of the SEIQoLDW led to greater variability.

(Continued on next page)

\footnotetext{
* Correspondence: almuth.berg@medizin.uni-halle.de

${ }^{1}$ Institute of Health and Nursing Science, Medical Faculty, Martin Luther

University Halle-Wittenberg, Halle (Saale), Germany

Full list of author information is available at the end of the article
}

(c) The Author(s). 2020 Open Access This article is licensed under a Creative Commons Attribution 4.0 International License, which permits use, sharing, adaptation, distribution and reproduction in any medium or format, as long as you give appropriate credit to the original author(s) and the source, provide a link to the Creative Commons licence, and indicate if changes were made. The images or other third party material in this article are included in the article's Creative Commons licence, unless indicated otherwise in a credit line to the material. If material is not included in the article's Creative Commons licence and your intended use is not permitted by statutory regulation or exceeds the permitted use, you will need to obtain permission directly from the copyright holder. To view a copy of this licence, visit http://creativecommons.org/licenses/by/4.0/. The Creative Commons Public Domain Dedication waiver (http://creativecommons.org/publicdomain/zero/1.0/) applies to the data made available in this article, unless otherwise stated in a credit line to the data. 
(Continued from previous page)

Conclusions: For cardiac surgery patients, use of the current version of the SEIQoL-PF/G as a substitute for the SEIQoLDW is not recommended.

The current questionnaire weighting method seems to be unable to distinguish weighting for different cues. Therefore, the further design of a weighting method without interviewer support as a paper-administered measure of individual quality of life is desirable.

Keywords: Quality of life, Patient reported outcome measures, Psychometrics, Clinical trials, Cardiac surgery

\section{Background}

Quality of life (QoL) as a patient-reported outcome has growing importance in clinical research [1]. A variety of QoL measures with different dimensions and underlying theoretical assumptions have been developed to assess QoL [2]. Nevertheless, no consensus exists regarding the essential domains of the QoL [3]. Traditional QoL measures often emphasize physical dimensions, although mental and social dimensions should be more prominent from a stakeholder perspective [3]. Attempts to incorporate individually relevant values and dimensions into QoL measurements by using more respondent-generated QoL measures to determine individuals' QoL have increased [4]. The Schedule for the Evaluation of Individual Quality of Life (SEIQoL) [5-7] is a measure that allows respondent-generated QoL dimensions. This measure is an interview-based assessment of QoL from an individual's perspective. In contrast to researcher-generated instruments that use predefined QoL dimensions, the SEIQoL offers the opportunity to nominate, weight, and rate dimensions that are considered important for QoL from an individual's unique perspective. However, this approach requires more time and effort during the data collection phase. Therefore, a short form of the instrument - the SEIQoL-Direct Weighting method (SEIQoL-DW) $[8,9]$ - was developed and used in numerous studies with a variety of populations $[10,11]$. Convergent and discriminant validity of the SEIQoL-DW can be described as moderate-to-high for global QoL, life satisfaction, and mental health but weak for the functional status and health measures [10].

The use of this short form has some pragmatic limitations in research, largely because it must be delivered in a personal semi-structured interview. Wettergren et al. [10] described mean administration times ranging from 5 to up to $50 \mathrm{~min}$ for one single SEIQoL-DW interview. This requirement limits the applicability and feasibility of this measure in research, because considerable resources for data collection are required. Therefore, some paper and computer adaptations of the SEIQoL-DW have been developed [12-16]. However, in a preliminary literature search, we were not able to identify any data regarding agreement between the original SEIQoL-DW and an adaption administered in written form.
Therefore, our study aims to explore the agreement between the SEIQoL-DW and a self-developed, paperadministered version of the SEIQoL-DW that assesses the following factors:

(1) agreement between the two SEIQoL indices;

(2) agreement between the cue levels in each cue; and

(3) agreement between the cue weights in each cue.

Our paper-based questionnaire was intended for postal use in a clinical trial and hereafter will be referred to as the Schedule for the Evaluation of Individual Quality of Life-Paper Form/German language (SEIQoL-PF/G). Since the person determines their own QoL concept, patient understanding of the draft instrument was evaluated according to the ISPOR recommendations [17] for content validity using cognitive interviews [18]. The feasibility of the resulting version was tested within a randomized controlled trial with patients in cardiac surgery, general surgery, and internal medicine [19].

\section{Methods}

We describe the methods and results of our study according to the Guidelines for Reporting Reliability and Agreement Studies (GRRAS) checklist [20].

\section{Study design}

The study was designed as a crossover trial in which both measures were completed by the patients in a random sequence as follows:

- Sequence group 1: first measurement SEIQoL-DW, second measurement SEIQoL-PF/G,

- Sequence group 2: first measurement SEIQoL-PF/G, second measurement SEIQoL-DW.

The rationale for choosing this study design was to control for effects of order of presentation in the completion of the instruments. 


\section{Measures \\ SEIQoL-DW}

The SEIQoL-DW is administered in a face-to-face interview. After an introduction of the QoL concept from the individual's perspective, the SEIQoL-DW is performed in three steps. First, the interviewee chooses the five individual aspects that are the most important areas of their life (cues). If the respondent cannot nominate five cues, a prompt list with examples is provided. The interviewer documents the meaning of each cue as labeled by the respondent (cue label). Second, the interviewee rates their current performance (cue level) in each area on a bar chart. Finally, the interviewee assigns weights to the nominated areas in relation to each other on a special weighting disc in the form of a pie chart (cue weight). During each stage, the interviewers provide comprehensive support through explanations and examples. The outcomes of the SEIQoL-DW are cue labels and their definitions, cue levels and cue weights. The levels are scored using the height of the bar charts (0 to 100), which yields five scores from independent continuous measurements. From the sum of the products of the levels and the relative weights of the five cues, an index can be calculated and used for comparisons on an individual or group level with a range from 0 to $100[8,9]$.

\section{SEIQOL-PF/G}

The SEIQoL-PF/G (see Additional file 1) is constructed as a paper questionnaire version in the German language and is self-explanatory for self-administration without support from an interviewer. The major modifications of the SEIQoL-DW include the addition of brief written instructions, a prompt list with examples of the most common cues, and an explanation of how to use the visual analogue scale (VAS) in the questionnaire. With the VAS, rating and weighting is conducted for each cue. The index is calculated by adding the products of the levels and the relative weights of the five cues. The relative weight of a cue is calculated by dividing the weight of the cue in the VAS by the sum of the weights of the VASs of all cues [18].

\section{Setting and participants}

The most common application of the SEIQoL-DW is the description of the QoL of patients with specific chronic or incurable diseases [10]. Because the feasibility of the SEIQoL-PF/G was previously tested with cardiac surgery patients [19], the study was conducted in a similar population between May 2013 and June 2013 in the Sana Heart Surgery Hospital in Stuttgart, Germany. The hospital covers the normal spectrum of cardiac surgery types. After surgery, patients are monitored for an average of one to 2 days in the intensive care unit. Once their condition is stable, they are transferred to a general ward. On average, patients are transferred to a rehabilitation facility after eight to ten days.

Patients were eligible for the study if they had completed surgery and, from a clinician' point of view, they were physically and cognitively able to fill out a questionnaire. Additional inclusion criteria were an absence for a minimum of $24 \mathrm{~h}$ of all signs of postoperative acute confusion routinely assessed with the Delirium Observation Screening Scale [21] and an age $>18$ years. Patients with diagnosed dementia or other known cognitive impairment were excluded from study participation.

\section{Sample size calculation}

For our primary analysis, there is currently no statistical procedure for a sample size calculation [22]. Thus, we used the intraclass correlation coefficient (ICC) to estimate the sample size. We wanted to obtain an ICC of at least $\rho=0.7$ with a $95 \%$ confidence interval $(\mathrm{CI})$ from 0.5 to 0.9 and $\mathrm{k}=2$ ratings per person. In accordance with Bonett [23], this calculation resulted in a total of $n=104$ participants.

\section{Measurement process}

Six study nurses who were familiar with heart surgery patients conducted the recruitment and data collection.

Patients with an extended stay at the intensive care unit or who had undergone surgery with a heart-lung machine were recruited by a study nurse on day 4 after surgery at the earliest, whereas all other patients were approached on day 1 after surgery at the earliest. If the patient felt unwell or the staff in charge had objections for any health or psychological reason, the measurement was postponed to the next day.

The randomization sequence was computer generated ( $\mathrm{R}$ Package blockrand, version 1.3) in advance by employing a stratified (by the rater, i.e., study nurses) balanced block randomization. Group allocation was concealed using sequentially numbered opaque sealed envelopes and took place immediately after informed consent was given and baseline data were collected. The participants were randomly assigned to receive either the SEIQoL-DW or the SEIQoL-PF/G as the first measurement in sequence. Immediately after allocation to a sequence group, the patients conducted the respective SEIQoL version either autonomously in the presence of the study nurse (SEIQoL-PF/G) or together with the study nurse (SEIQoL-DW). The study nurses received special training with the SEIQoL-DW instructions [9].

To enable statistical analyses of the agreement, the nominated cues at the first measurement were transferred to the subsequent SEIQoL version.

The other version of the SEIQoL was completed at some point during the following days provided that the patient remained sufficiently stable. We selected a period 
of 1 to 4 days between the two consecutive measurements to balance between (i) avoiding period effects (which may arise because of potential changes in the mental or health status of the patient), and (ii) eliminating memory effects (which we assumed would bias the results toward better agreement).

Efforts were undertaken to standardize the measurement situation and to minimize the influence of external circumstances. The measurements were conducted in separate rooms. If that was not possible, the study nurse ensured a situation out of sight and out of earshot of other persons. Each patient conducted both measures with the same study nurse.

Data for the study sample description were extracted from the patients' medical records.

\section{Statistical analysis}

As recommended for metric variables [22, 24], agreement between the two SEIQoL indices was evaluated with Bland-Altman plots, in which the difference between the two sequential measures was plotted against their mean value. Bland-Altman plots allow the interpretation of the extent of differences. Therefore, if limits of agreement (LoAs) are computed, 95\% of the sample differences will lie between these limits. Additionally, the 95\% CIs of the mean difference and the LoAs were calculated.

To interpret the LoAs, we tried to define a threshold for the determination of their acceptability. Because differences within the scope of half of a standard deviation were considered noticeable in QoL measures [25], and a standard deviation of 20 scale points was applied in a recent study [19], we determined that a difference of 10 scale points was a meaningful threshold. This approach means that to achieve acceptable agreement, the outside limits of the CI of the LoA must be within this range.

Additionally, the ICC of the two indices and its CI was calculated [23] to describe the intraclass correlation.

Bland-Altman plots were also constructed for the cue levels and cue weights, although we did not predefine thresholds.

The effects of instruments or the sequence order as well as the interaction of both of these factors were estimated in a linear mixed model (random intercept only) with corresponding 95\% CIs using the R Package nlme, version 3.1-109.

\section{Quality assurance}

Data collection and processing were conducted using pseudonyms. Data entry and monitoring were performed by one researcher $(\mathrm{MB})$ within 3 days of data collection. Any irregularities were clarified immediately with the study staff.

\section{Results}

During the study period, a total of 166 patients were assessed for eligibility (see Fig. 1), of which we recruited 104 patients. Five patients in sequence group 2 (first measurement: SEIQoL-PF/G) discontinued study participation. Overall, data from $n=99$ patients were used to estimate the agreement between the instruments.

The average time between the two measurements was 1 day (mean 1.3; SD 0.8).

\section{Study sample description}

Table 1 provides the characteristics of the study participants with both measures grouped by sequence. A slight difference was found only for the type of surgery, because an imbalance existed between the numbers of surgical valve replacements and coronary bypass grafts. However, both procedures are comparable in terms of the postoperative effects on the patient. Sequence group 2 included fewer patients with independent mobility and had a higher rethoracotomy rate due to complications. However, analysis of the subgroup with rethoracotomy showed no deviations in the sociodemographic or clinical data.

The participants included in this study reflect characteristic features and types of surgical procedures of other heart surgery hospitals in Germany [26].

\section{Comparison of the SEIQoL-DW and SEIQoL-PF/G for the nominated cues}

All patients nominated five cues at the first measurement (sequence group 1: SEIQoL-DW, $n=52$; sequence group 2: SEIQoL-PF/G, $n=49$ ).

Thus, the group performing the SEIQoL-DW first nominated $260(52 \times 5)$ cues, and the other group named $245(49 \times 5)$ cues. With few exceptions, no substantial differences were found in the nature and frequency of the cues (see Table 2). Only the cue "religion/spiritual life" was nominated more frequently with the SEIQoLDW, whereas the cue "travel" was clearly favored with the SEIQoL-PF/G. With both instruments, more than $50 \%$ of the patients nominated the areas of life "physical health", "family", and "partnership".

The associated rating of levels was heterogeneous for all cues (see Table 2). The considerable range indicated that some patients tended to rate in the margins of the scale. Only one significant difference was found for the cue "sports", which was rated higher with the SEIQoL$\mathrm{PF} / \mathrm{G}$ than with the SEIQoL-DW.

Considering the associated weighting process, differences between the SEIQoL-DW and SEIQoL-PF/G became obvious, especially given the dispersion of the data; the SEIQoL-DW group showed substantially greater dispersion than the SEIQoL-PF/G group (see Table 2). Although the SEIQoL-DW cues were different in terms of 


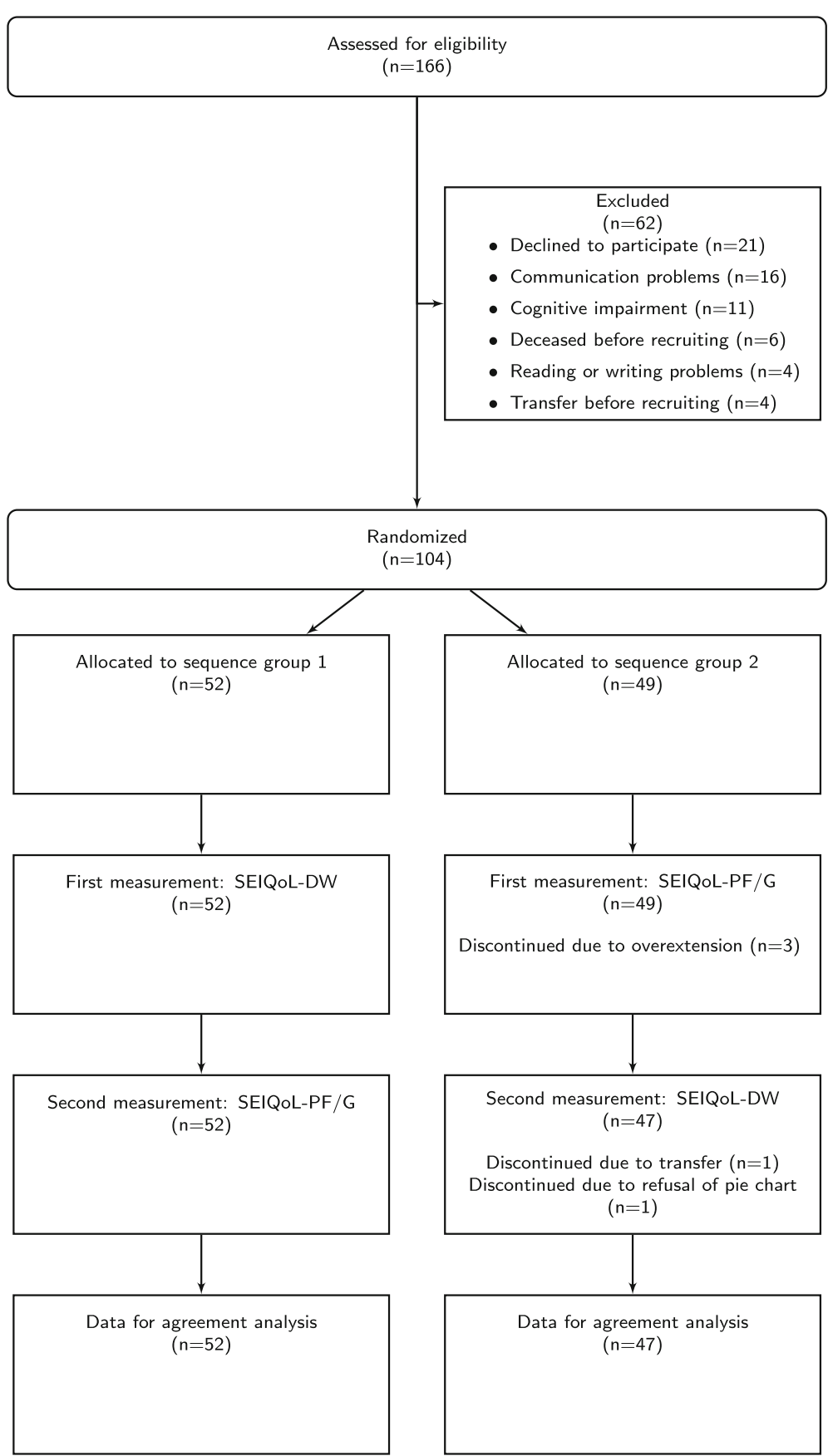

Fig. 1 Flow chart of the study participants

weighting, the SEIQoL-PF/G cues appeared to be nearly equivalent. The cues "physical health" and "partnership" were weighted significantly lower with the SEIQoL-PF/ G, whereas the cues "friends", "nature/garden", "sports", and "finances" showed significantly higher values with the SEIQoL-PF/G.

The SEIQoL-DW group had a mean index of 70.0 $(\mathrm{SD}=17.1)$ compared to a mean index of $70.3(\mathrm{SD}=$
16.8) for the SEIQoL-PF/G group [mean difference $=0.4$ (95\% CI, -6.3 to 7.1$)$ ].

\section{Agreement between the SEIQoL-DW and SEIQoL-PF/G}

The agreement between the indices of the two instruments is shown in the Bland-Altman plot (see Fig. 2). The mean of the differences was 2 index points $(95 \% \mathrm{CI}$, - 1 to 6). The upper LoA showed a difference of 36 
Table 1 Characteristics of the study participants with both measures

\begin{tabular}{|c|c|c|c|}
\hline & $\begin{array}{l}\text { Sequence group } 1 \\
n=52\end{array}$ & $\begin{array}{l}\text { Sequence group } 2 \\
n=47\end{array}$ & $\begin{array}{l}\text { Total } \\
n=99\end{array}$ \\
\hline Age/years, mean (SD) & $68.0(12.0)$ & $65.0(14.3)$ & $66.6(13.6)$ \\
\hline \multicolumn{4}{|l|}{ Gender, n (\%) } \\
\hline Male & $40(76.9)$ & $35(74.5)$ & $75(75.8)$ \\
\hline \multicolumn{4}{|l|}{ Mode of hospital admission, n (\%) } \\
\hline Elective & $44(84.6)$ & $38(80.9)$ & $82(82.8)$ \\
\hline Emergency & $8(15.4)$ & $9(19.1)$ & $17(17.2)$ \\
\hline \multicolumn{4}{|l|}{ Surgical procedure, n (\%) } \\
\hline Coronary bypass crafting & $20(38.5)$ & $13(27.7)$ & $33(33.3)$ \\
\hline Surgical valve replacement & $13(25.0)$ & $16(34.0)$ & $29(29.3)$ \\
\hline Both procedures & $7(13.5)$ & $8(17.0)$ & $15(15.2)$ \\
\hline Other valve surgery & $9(17.3)$ & $8(17.0)$ & $17(17.2)$ \\
\hline Other procedure & $3(5.8)$ & $2(4.3)$ & $5(5.1)$ \\
\hline Length of ICU stay/days, mean (SD) & $2.0(1.5)$ & $2.7(3.1)$ & $2.3(2.4)$ \\
\hline Acute confusion in the $\mathrm{ICU}^{\mathrm{a}}, \mathrm{n}(\%)$ & $7(13.5)$ & $7(14.9)$ & $14(14.1)$ \\
\hline Postoperative complications: rethoracotomy, n (\%) & $1(1.9)$ & $5(10.6)$ & $6(6.1)$ \\
\hline Days after surgery at the first SEIQoL measurement, mean (SD) & $5.2(2.6)$ & $6.1(4.6)$ & $5.6(3.7)$ \\
\hline \multicolumn{4}{|l|}{ Mobility status at the first SEIQoL measurement, n (\%) } \\
\hline Mobility in bed & 0 & 0 & 0 \\
\hline Mobility up to edge of bed & $1(1.9)$ & $4(8.5)$ & $5(5.0)$ \\
\hline Mobility to chair & $7(13.5)$ & $9(19.1)$ & $16(16.2)$ \\
\hline Walking with assistance & $11(21.2)$ & $11(23.4)$ & $22(22.2)$ \\
\hline Independent mobility & $33(63.5)$ & $23(48.9)$ & $56(56.6)$ \\
\hline
\end{tabular}

${ }^{a}$ Routinely assessed with the Delirium Observation Screening Scale

index points ( $95 \% \mathrm{CI}, 30$ to 42 ), and the lower LoA showed a difference of -31 index points $(95 \% \mathrm{CI},-37$ to -26). Thus, even in the best case, both LoAs and their CIs were far larger than the predefined tolerable threshold of 10 scale points.

The deviations were also larger if the mean index values ranged between 55 and 75 . This result can be mainly explained by the nature of a scale that is calculated as a mean of two scales with inherent scale limits (0 and 100), which results in lower possible differences adjacent to the borders of the mean scale. Given the unambiguous overall result, we did not further analyze any of the outliers.

The ICC that was used to quantify the dependency of both group index values at the two time points indicated a medium correlation: $\rho=0.49$ (95\% CI, 0.32 to 0.62 ).

The agreement of the cue rating is shown in Fig. 3. The mean difference was 6 scale points (95\% CI, 4 to 8), and the LoAs ranged between $-47(95 \% \mathrm{CI},-51$ to $43)$ and 58 (95\% CI, 54 to 63).

The cue rating represents a very large deviation considering a range between 0 and 100. The narrow CIs, which span less than 10 points, indicate a very accurate measure. The agreement is better in the margin areas of the scale than in the middle range, which can be explained by the same mechanism as that applicable for the index. The reduced variability observed here also indicated that the patients often rated their satisfaction in the border areas. This result was similar with both instruments.

The agreement of the weighting values is shown in Fig. 4. The mean difference was 0 (95\% CI, 0 to 1 ), with LoAs between 19 (95\% CI, 17 to 21) and -19 (95\% CI, -21 to -17 ).

Considering that the weight value of $100 \%$ is divided between 5 cues with both instruments and that most of the patients weighted their cues below $30 \%$, a nearly $20 \%$ deviation is considerable. Moreover, in this plot, a linear relationship that indicates a systematic difference between the instruments is visible. In the weighting range between 5 and 17 scale points, the SEIQoL-DW rated values lower than the SEIQoL-PF/G. Proceeding upwards from 25 scale points, lower weights were given with the SEIQoL-PF/G. This result shows a tendency toward equal weighting of cues in the SEIQoL-PF/G version, while the weighting procedure of the SEIQoL-DW 
Table 2 Comparison of the SEIQoL-DW and SEIQoL-PF/G for the nominated cues collected at the first measurement (SEIQoL-DW: $n=52$, SEIQoL-PF/G: $n=49$ )

\begin{tabular}{|c|c|c|c|c|c|c|}
\hline \multirow[t]{2}{*}{ Nominated cues $^{a}$} & \multicolumn{3}{|c|}{ Rating level: satisfaction } & \multicolumn{3}{|c|}{ Relative weight: importance } \\
\hline & $\begin{array}{l}\text { SEIQOL-PF/G, } \\
\text { mean (SD) }\end{array}$ & $\begin{array}{l}\text { SEIQoL-DW, } \\
\text { mean (SD) }\end{array}$ & Mean difference $(95 \% \mathrm{Cl})$ & $\begin{array}{l}\text { SEIQOL-PF/G, } \\
\text { mean (SD) }\end{array}$ & $\begin{array}{l}\text { SEIQoL-DW, } \\
\text { mean (SD) }\end{array}$ & Mean difference $(95 \% \mathrm{Cl})$ \\
\hline Physical health & $63.3(23.0)$ & $54.95(32.8)$ & $8.3(-4.2 ; 20.8)$ & $21.6(2.5)$ & $25.7(11.9)$ & $-4.1(-7.9 ;-0.3)$ \\
\hline$n=80$ & $n=38$ & $n=42$ & & & & \\
\hline Family & $80.3(21.4)$ & $83.5(14.2)$ & $-3.2(-11.7 ; 5.3)$ & $22.6(4.0)$ & $25.5(8.9)$ & $-3.0(-6.0 ; 0.1)$ \\
\hline$n=78$ & $n=34$ & $n=44$ & & & & \\
\hline Partnership & $83.5(17.8)$ & $85.0(20.5)$ & $-1.5(-11.5 ; 8.5)$ & $21.6(3.1)$ & $26.7(10.8)$ & $-5.0(-9.3 ;-0.8)$ \\
\hline$n=59$ & $n=30$ & $n=29$ & & & & \\
\hline Friends & $71.5(18.1)$ & $75.0(16.7)$ & $-3.5(-13.9 ; 6.9)$ & $19.7(1.5)$ & $15.6(5.9)$ & $4.1(1.5 ; 6.6)$ \\
\hline$n=46$ & $n=22$ & $n=24$ & & & & \\
\hline Work/occupation & $60.1(28.5)$ & $61.4(35.3)$ & $-1.3(-21.4 ; 18.9)$ & $18.6(4.3)$ & $18.7(10.9)$ & $-0.1(-5.2 ; 5.1)$ \\
\hline$n=41$ & $n=19$ & $n=22$ & & & & \\
\hline Nature/garden & $70.3(29.1)$ & $56.1(31.6)$ & $14.2(-9.4 ; 37.7)$ & $18.8(2.2)$ & $13.2(6.2)$ & $5.6(2.0 ; 9.2)$ \\
\hline$n=28$ & $n=13$ & $n=15$ & & & & \\
\hline Sports & $60.7(29.8)$ & $27.2(25.9)$ & $33.5(10.8 ; 56.1)$ & $19.1(2.7)$ & $12.3(6.0)$ & $6.9(2.7 ; 11.0)$ \\
\hline$n=26$ & $n=15$ & $n=11$ & & & & \\
\hline Finances & $66.6(14.9)$ & $66.5(16.2)$ & $0.1(-19.7 ; 13.9)$ & $17.9(2.7)$ & $12.2(5.8)$ & $5.7(1.4 ; 10.1)$ \\
\hline$n=23$ & $n=13$ & $n=10$ & & & & \\
\hline Travel & $58.2(29.7)$ & $60.7(36.9)$ & $-2.4(-82.8 ; 78.0)$ & $17.6(3.7)$ & $14.3(5.1)$ & $3.3(-8.1 ; 14.7)$ \\
\hline$n=19$ & $n=16$ & $n=3$ & & & & \\
\hline Hobbies & $60.7(19.1)$ & $49.9(18.4)$ & $10.8(-8.0 ; 29.5)$ & $17.8(2.4)$ & $14.2(9.3)$ & $3.6(-3.7 ; 10.8)$ \\
\hline$n=18$ & $n=9$ & $n=9$ & & & & \\
\hline Food/beverage & $60.4(25.9)$ & $69.9(23.9)$ & $-9.5(-37.3 ; 18.3)$ & $19.1(3.1)$ & $14.6(9.1)$ & $4.6(-4.0 ; 13.1)$ \\
\hline$n=15$ & $n=8$ & $n=7$ & & & & \\
\hline Leisure activities & $69.6(26.3)$ & $56.1(34.1)$ & $13.5(-23.9 ; 50.9)$ & $18.6(2.1)$ & $16.1(8.8)$ & $2.5(-5.0 ; 10.0)$ \\
\hline$n=13$ & $n=5$ & $n=8$ & & & & \\
\hline Psychological health & $64.8(30.8)$ & $69.8(23.5)$ & $-5.0(-42.0 ; 32.1)$ & $17.3(4.5)$ & $19.6(5.7)$ & $-2.3(-9.6 ; 5.0)$ \\
\hline$n=11$ & $n=6$ & $n=5$ & & & & \\
\hline Religion/spiritual life & $69.0(--)$ & $76.8(26.6)$ & $-7.8(--)$ & $19.0(--)$ & $20.1(6.9)$ & $-1.1(--)$ \\
\hline$n=10$ & $n=1$ & $n=9$ & & & & \\
\hline
\end{tabular}

${ }^{a}$ Cues that were mentioned by at least $10 \%$ of all patients

leads to greater variability. An additional analysis of the box plots of the weighted cues of both sequence groups supports this finding (data not shown).

Overall, lower variation exists in the weighting as in the indices or at the cue levels.

No significant instruments or sequence order effects were found (i.e., the results were not time dependent; data not shown).

\section{Discussion}

The aim of our study was to explore the agreement between the SEIQoL-DW and the paper-administered version (SEIQoL-PF/G) in terms of the indices, cue levels, and cue weights.
Our analysis clearly shows a substantial lack of agreement between the SEIQoL-DW and SEIQoL-PF/G. The lack of agreement between the indices became especially evident in our graphic analysis with the Bland-Altman plot. The LoAs in this plot show that the measured index value differences are far higher than the predefined limit of 10 index points.

Due to missing statistical methods for a sample size calculation for this evaluation method, we used the ICC. Because of this provisional nature, sample size was not further adjusted such as an expected loss to follow-up. The ICC of the two indices shows a medium intraclass correlation. Even though this is based on $n=5$ patients less than in the sample size calculation, the confidence 


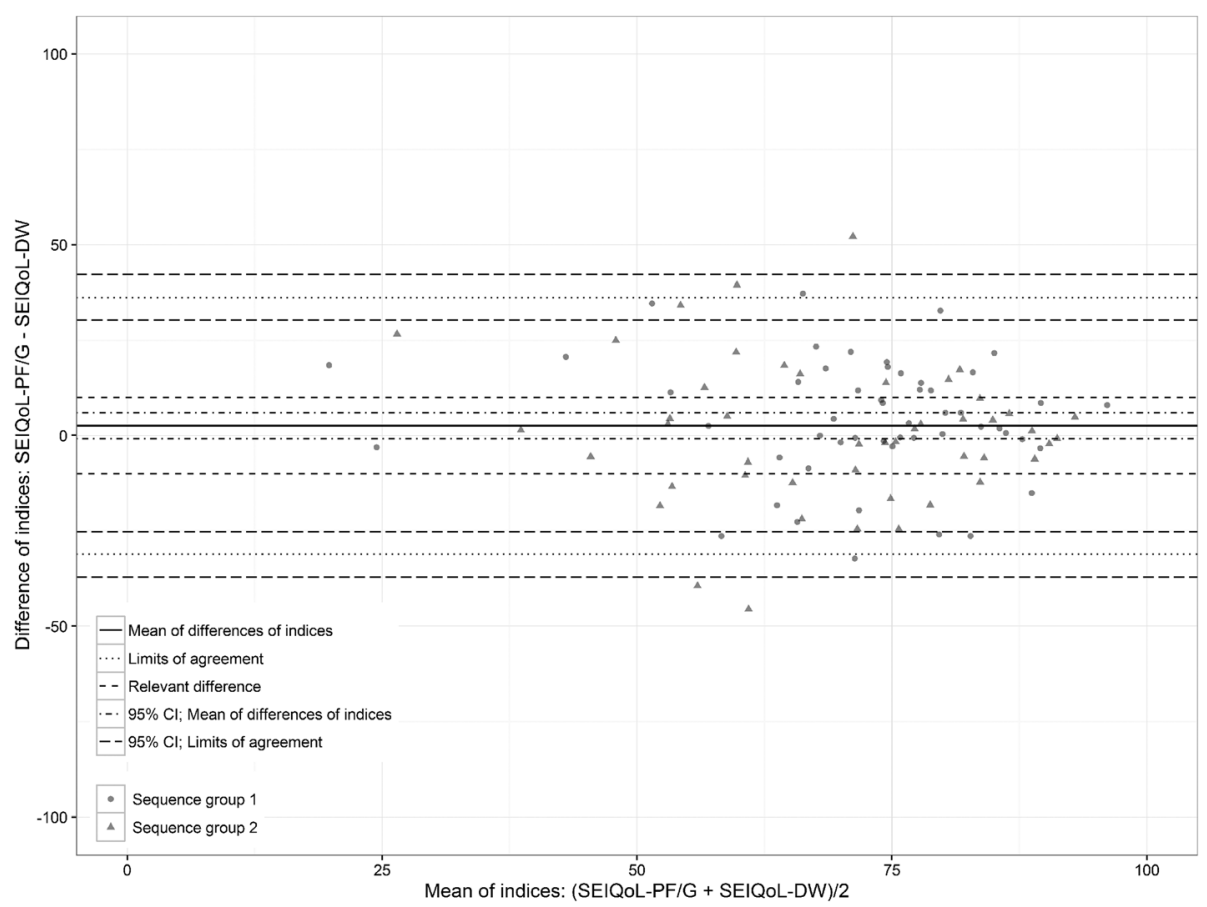

Fig. 2 Bland-Altman plot of differences in index measures obtained with the SEIQOL-DW and SEIQOL-PF/G

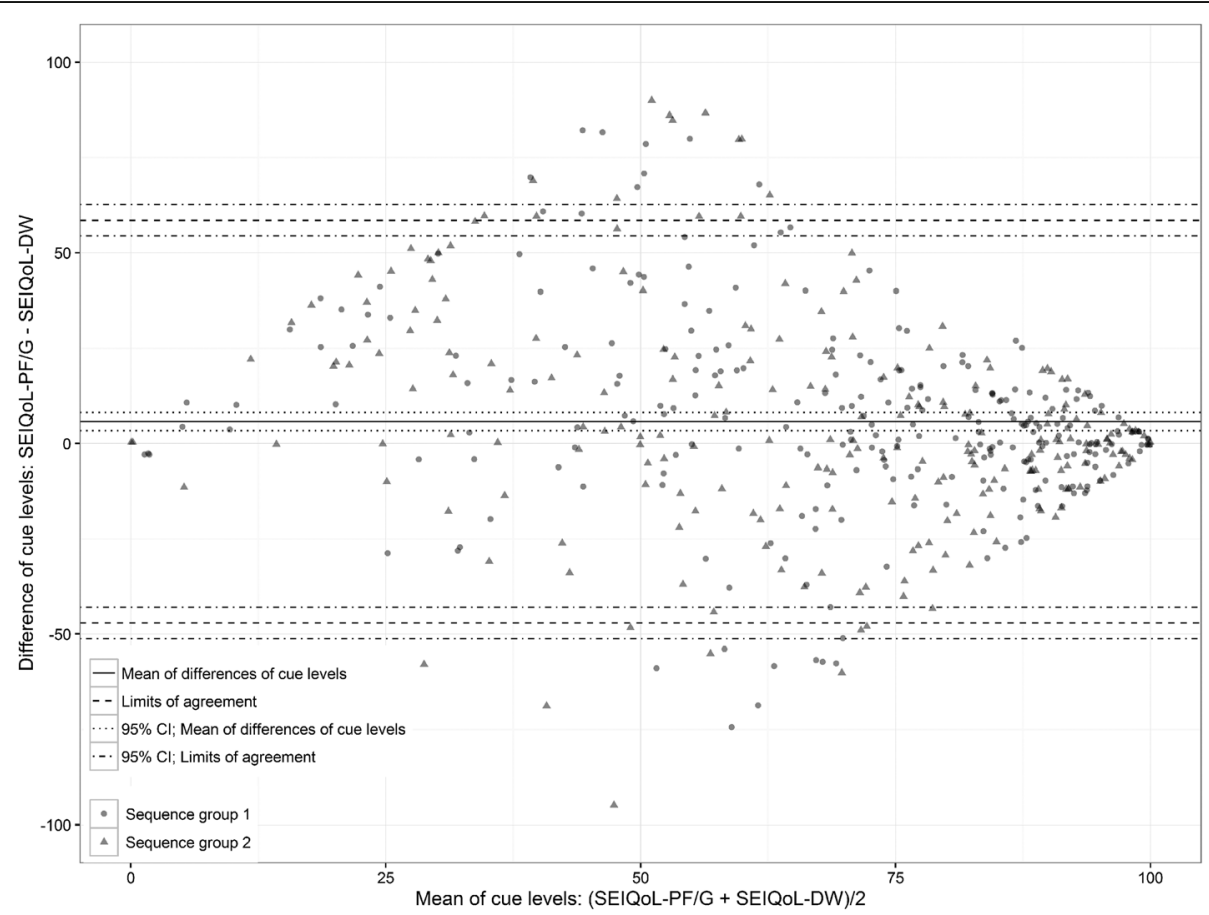

Fig. 3 Bland-Altman plot of differences in the cue levels measured with the SEIQoL-DW and SEIQoL-PF/G 


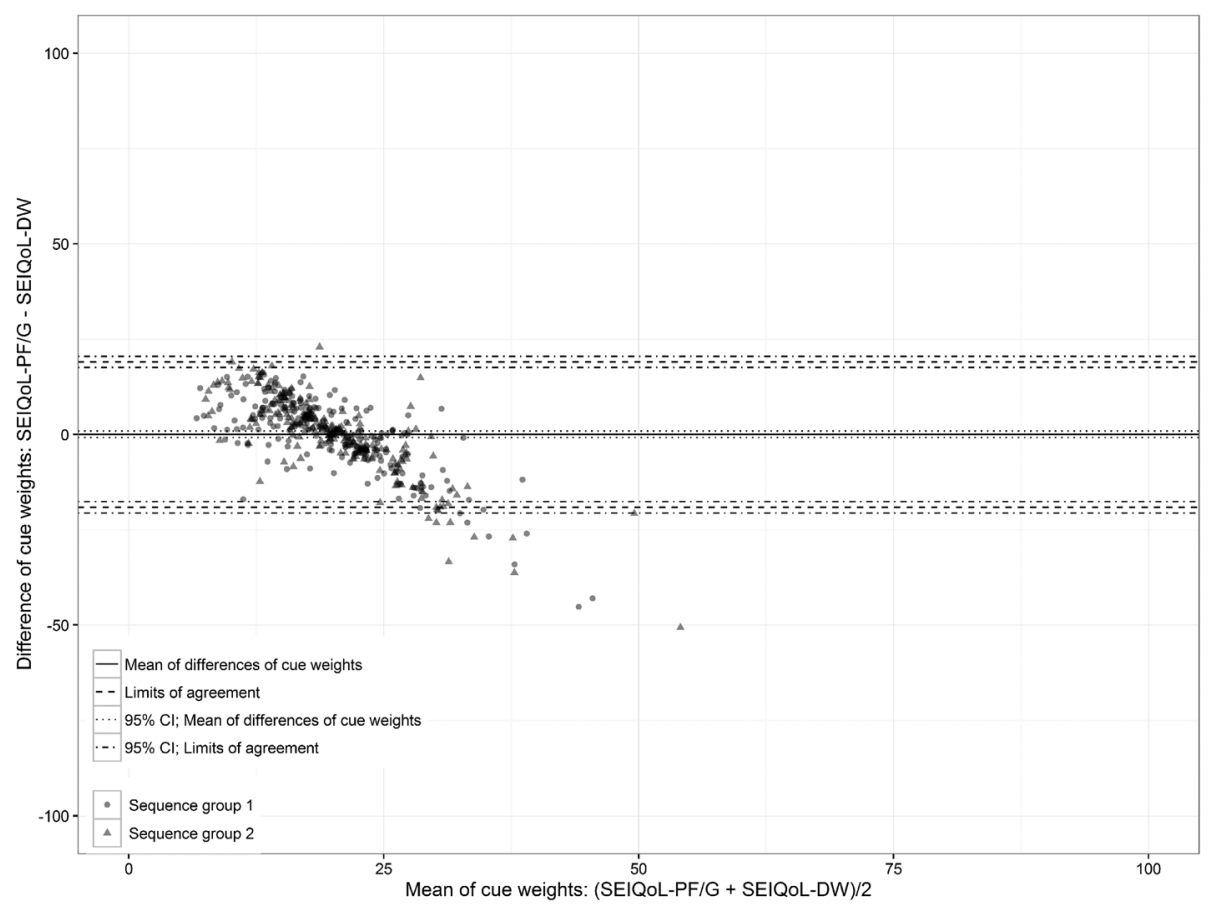

Fig. 4 Bland-Altman plot of differences in the weights measured with the SEIQOL-DW and SEIQoL-PF/G

interval, as this is clearly below the original assumptions, implies that it is not likely to have missed a large correlation. However, correlation coefficients suggest a linear relationship of two metric variables, and they should not be used as an agreement assessment [24, 27]. Considering the clear results, we do not expect to achieve better agreement of the two measures in future studies.

This finding is also true for the other Bland-Altman plots in our analyses constructed for cue levels and cue weights. The latter even revealed a systematic difference between the two weighting methods, whereas the other deviations implied random variation of the measured values.

The data of both measures collected for the nominated cues at the first measurement (Table 2) confirm these results.

Both instruments led to similarly nominated cues. By far the most frequently nominated cues were "physical health" and "family". The cues "family" and "health" were also among the most relevant domains in similar cardiac patient groups in both the SEIQoL-DW $[28,29]$ and a paper-administered version $[12,18]$.

The rating of cues (Table 2) was heterogeneous, including one significant difference in the cue "sports", which had higher ratings in the SEIQoL-PF/G. Interviewer comments revealed that this discrepancy was due to different meanings attached to the cue "sports", such as "watching sports on TV" and "playing sports". In accordance with the SEIQoL-DW manual, the interviewers tried to capture the correct meaning when "sports" was rated very high, even if the patient was unable to engage in physical activity a few days after surgery.

For the weighting procedure, the SEIQoL-DW data indicate a relationship between frequent cue nomination and high weighting (Table 2). In contrast, most of the weightings of the SEIQoL-PF/G were nearly equivalent within a narrow range of 20 scale points. Considering the systematic difference found in the Bland-Altman plot, these findings suggest that the SEIQoL-PF/G weighting method is unable to clearly distinguish important from less important cues. Probably, the five separately listed VASs cannot be sufficiently distinguished in terms of their relationships to each other to achieve relative weighting.

However, the mean indices of the SEIQoL-DW and SEIQoL-PF/G group (70.0 vs. 70.3) seem to be unaffected by the deviations in the rating and weighting (shown in Table 2) and show similar values. The SEIQoL-PF/G mean index is considered questionable due to the indifferent weighting method. The SEIQoLDW mean index is slightly lower (representing a lower quality of life) than that in a comparable setting with cardiac patients [28]. In that study, the index was 81 but referred to patients with minor surgical procedures.

Overall, we can assume that the current SEIQoL-PF/G version is not interchangeable with the SEIQoL-DW as a valid substitute. Nevertheless, a paper-administered measure of individual QoL has great pragmatic 
importance for use in clinical trials. In our study, the weighting method of the current SEIQoL-PF/G version proved to be problematic. Therefore, the modification of the measure in terms of further development of a differentiating weighting method without interviewer support is desirable. Although the SEIQoL-DW has been explicitly developed for use in clinical practice [7], a more pragmatic version could also be very useful as a tool for care management of patients in clinical practice settings. In particular, identifying areas that are especially important to the individual patient, may support care planning and clinical decision-making [10].

\section{Strengths and limitations}

We assessed agreement between two measures in a randomized crossover design with the concealed allocation of participants to the sequence groups. With a randomized sequence order, it was possible to avoid effects of order of instrument presentation, because we did not know a priori whether the order of completion could have an influence on the results. This procedure corresponds to the recommendations of testing adaptations for agreement with original instruments [30].

Because no validated German translation of the SEIQoL-DW manual existed, it had to be done by the project team. However, these are rather instructions for the study nurses to perform the semi-structured interview than the administration of a questionnaire. The instructions were provided to all study nurses and they received a special training.

The results may be influenced by the time span (mean: 1 day) between the completions of the two versions. Concerning an appropriate period to avoid potential memory effects, we could not find recommendations. Over a 1-week period, however, weaknesses in the testretest reliability of the SEIQoL-DW in healthy individuals were found [31]. Therefore, to avoid both period effects by health status changes of the patient (from a clinical point of view) and memory effects to the first version, we determined a period of 1 to 4 days. Factors such as emotional states or daytime-dependent variables that could have changed the participants' judgment could not be assessed. Thus, a clinically founded change in rating or weighting of cues cannot be excluded with certainty. However, we took precautions not to interview patients who felt unwell or had any indication of instability.

Another limitation arises for the interpretation of the ICC in our study. This limitation is associated with the nature of the SEIQoL concept that calculates the score as a multiplicative composite. That means the score is derived from a multiplication of two components: cue rating and cue weighting. The scaling of these variables can have a tremendous impact on correlation with an external measure [32, 33]. A simple linear transformation of the variables can reduce the coefficient or even change the direction of the association [32, 33]. Therefore, the ICC has to be interpreted with caution. However, the ICC is only a secondary indicator in our study; the main analysis is based on the Bland-Altman plots. An approach in future analyses focussing on ICC should apply hierarchical regression modeling [32].

Generally, the studied population was very specific and included only cardiac surgery patients. In that, other populations (e.g. other diseases, healthy population) may lead to different results. However, our study provides valid reference data for acceptable agreement ranges for future research with the SEIQoL-DW. Additionally, the represented main cues embody the perspective of cardiac patients and thus provide a useful adjunct for clinical and research-related issues in this patient group.

\section{Conclusion}

According to our findings, this study is the first to analyze agreement between the original SEIQoL-DW and a version administered without interviewer support. Our data indicate an unacceptable range of agreement between the SEIQoL-DW and the paper-based questionnaire SEIQoL-PF/G for cardiac surgery patients. Thus, use of the current version of the SEIQoL-PF/G as a substitute for the SEIQoL-DW in clinical practice or research is not recommended.

\section{Supplementary information}

Supplementary information accompanies this paper at https://doi.org/10. 1186/s12874-020-00961-9.

Additional file 1. SEIQOL-PF/G

\section{Abbreviations}

Cl: Confidence interval; ICC: Intraclass correlation coefficient (denoted as $\rho$, "rho"); LoA: Limit of agreement; SD: Standard deviation; SEIQoL: Schedule for the Evaluation of Individual Quality of Life; SEIQoL-DW: Schedule for the Evaluation of Individual Quality of Life-Direct Weighting; SEIQoL-PF/ G: Schedule for the Evaluation of Individual Quality of Life-Paper Form/German language; QoL: Quality of life; VAS: Visual analogue scale

\section{Acknowledgments}

Not applicable.

\section{Authors' contributions}

$A B$ and SF defined the study design. MB conducted the study. MB and SF were responsible for the statistical analysis. $A B$ provided substantial scientific advice, and SF supervised the study. All authors were responsible for the conception and design of the final manuscript. MB drafted the manuscript supported by $A B$ and SF. All authors read and approved the final manuscript.

\section{Funding}

The study was not funded.

We acknowledge the financial support of the Open Access Publication Fund of the Martin Luther University Halle-Wittenberg. 


\section{Availability of data and materials}

The dataset analyzed during the study is available from the authors upon reasonable request.

\section{Ethics approval and consent to participate}

Ethical approval for the study was obtained from the ethics committee of the Martin Luther University Halle-Wittenberg, Halle (Saale), Germany (approval reference: 2013-25)

Both written and verbal consent was obtained from all patients prior to inclusion in the study.

\section{Consent for publication}

Not applicable.

\section{Competing interests}

The authors declare that they have no competing interests.

\section{Author details}

${ }^{1}$ Institute of Health and Nursing Science, Medical Faculty, Martin Luther University Halle-Wittenberg, Halle (Saale), Germany. ${ }^{2} \mathrm{SRH}$ University of Applied Health Sciences, Gera, Germany.

Received: 15 October 2018 Accepted: 30 March 2020

Published online: 10 April 2020

\section{References}

1. Patrick DL, Guyatt GH, Acquadro C. Chapter 17: Patient-reported outcomes. In: Higgins JPT, Green S, editors. Cochrane handbook for systematic reviews of interventions, Version 5.1.0. The Cochrane Collaboration; 2011. Available from www.handbook.cochrane.org.

2. Carr AJ, Thompson PW, Kirwan JR. Quality of life measures. Br J Rheumatol. 1996:35(3):275-81.

3. Pietersma S, de Vries M, van den Akker-van Marle ME. Domains of quality of life: results of a three-stage Delphi consensus procedure among patients, family of patients, clinicians, scientists and the general public. Qual Life Res. 2014;23(5):1543-56.

4. Macduff C. Respondent-generated quality of life measures: useful tools for nursing or more fool's gold? J Adv Nurs. 2000;32(2):375-82.

5. McGee HM, O'Boyle CA, Hickey A, O'Malley K, Joyce CR. Assessing the quality of life of the individual: the SEIQOL with a healthy and a gastroenterology unit population. Psychol Med. 1991;21(3):749-59.

6. O'Boyle CA, McGee H, Hickey A, O'Malley K, Joyce CR. Individual quality of life in patients undergoing hip replacement. Lancet. 1992;339(8801):1088-91.

7. O'Boyle CA, McGee H, Hickey A, Joyce CRB, O'Malley K, Hiltbrunner B. The Schedule for the Evaluation of Individual Quality of Life (SEIQoL). Administration manual. Dublin: Royal College of Surgeons in Ireland; 1993. p. 1-73.

8. Hickey AM, Bury G, O'Boyle CA, Bradley F, O'Kelly FD, Shannon W. A new short form individual quality of life measure (SEIQOL-DW): application in a cohort of individuals with HIV/AIDS. BMJ (Clinical research ed). 1996; 313(7048):29-33.

9. O'Boyle C, Browne J, Hickey A, McGee HM, Joyce CRB. Schedule for the Evaluation of Individual Quality of Life (SEIQoL): a direct weighting procedure for quality of life domains (SEIQoL-DW). Administration manual. Dublin: Department of Psychology, Royal College of Surgeons in Ireland; 1995. p. 1-9.

10. Wettergren L, Kettis-Lindblad A, Sprangers M, Ring L. The use, feasibility and psychometric properties of an individualised quality-of-life instrument: a systematic review of the SEIQoL-DW. Qual Life Res. 2009;18(6):737-46.

11. Aburub AS, Mayo NE. A review of the application, feasibility, and the psychometric properties of the individualized measures in cancer. Qual Life Res. 2017;26(5):1091-104

12. Smith HJ, Taylor R, Mitchell A. A comparison of four quality of life instruments in cardiac patients: SF-36, QLI, QLMI, and SEIQoL. Heart. 2000; 84(4):390-4.

13. Ring L, Kettis-Lindblad A, Bendtsen P, Viklund E, Jansson R, Glimelius B. Feasibility and validity of a computer administered version of SEIQOL-DW. Qual Life Res. 2006;15(7):1173-7.

14. de la Rie S, Noordenbos G, Donker M, van Furth E. The patient's view on quality of life and eating disorders. Int J Eat Disord. 2007:40(1):13-20.
15. Kettis-Lindblad A, Ring L, Widmark E, Bendtsen P, Glimelius B. Patients' and doctors' views of using the schedule for individual quality of life in clinical practice. J Support Oncol. 2007;5(6):281-7.

16. Becker G, Merk CS, Meffert C, Momm F. Measuring individual quality of life in patients receiving radiation therapy: the SEIQoL-questionnaire. Qual Life Res. 2014;23(7):2025-30.

17. Patrick DL, Burke LB, Gwaltney CJ, Leidy NK, Martin ML, Molsen E, Ring L. Content validity: establishing and reporting the evidence in newly developed patient-reported outcomes (PRO) instruments for medical product evaluation: ISPOR PRO Good Research Practices Task Force report: part 2 - assessing respondent understanding. Value Health. 2011;14(8):97888.

18. Becker R, Berg A, Fleischer S, Neubert TR. Messung patientengenerierter Lebensqualität mittels schriftlicher Befragung - Entwicklung und erste Ergebnisse in der schriftlichen Anwendung des SEIQoL. Hallesche Beiträge zu den Gesundheits- und Pflegewissenschaften. 2009;8(4):3-15.

19. Fleischer S, Berg A, Behrens J, Kuss O, Becker R, Horbach A, Neubert TR. Does an additional structured information program during the intensive care unit stay reduce anxiety in ICU patients? A multicenter randomized controlled trial. BMC Anesthesiol. 2014;14(1):48.

20. Kottner J, Audige L, Brorson S, Donner A, Gajewski BJ, Hróbjartsson A, Roberts C, Shoukri M, Streiner DL. Guidelines for Reporting Reliability and Agreement Studies (GRRAS) were proposed. J Clin Epidemiol. 2011;64(1):96106.

21. Schuurmans MJ, Shortridge-Baggett LM, Duursma SA. The Delirium Observation Screening Scale: a screening instrument for delirium. Res Theory Nurs Pract. 2003;17(1):31-50.

22. Bland JM, Altman DG. Statistical methods for assessing agreement between two methods of clinical measurement. Lancet. 1986;1(8476):307-10.

23. Bonett DG. Sample size requirements for estimating intraclass correlations with desired precision. Stat Med. 2002;21:1331-5.

24. Altman DG. Assessing new methods of clinical measurement. Br J Gen Pract. 2009:59:399-400.

25. Norman GR, Sloan JA, Wyrwich KW. Interpretation of changes in healthrelated quality of life: the remarkable universality of half a standard deviation. Med Care. 2003;41(5):582-92.

26. Beckmann A, Funkat AK, Lewandowski J, Frie M, Ernst M, Hekmat K, et al. German Heart Surgery Report 2016: the annual updated registry of the German Society for Thoracic and Cardiovascular Surgery. Thorac Cardiovasc Surg. 2017;65(7):505-18.

27. Bland JM, Altman DG. A note on the use of the intraclass correlation coefficient in the evaluation of agreement between two methods of measurement. Comput Biol Med. 1990;20(5):337-40.

28. Gribbin GM, Kenny RA, McCue P, Toff WD, Bexton RS, McComb JM. Individualised quality of life after pacing. Does mode matter? Europace. 2004;6(6):552-60.

29. Dempster M, Carney R, McClements R. Response shift in the assessment of quality of life among people attending cardiac rehabilitation. $\mathrm{Br} J$ Health Psychol. 2010;15(Pt 2):307-19.

30. Coons SJ, Gwaltney CJ, Hays RD, Lundy JJ, Sloan JA, Revicki DA, et al. Recommendations on evidence needed to support measurement equivalence between electronic and paper-based patient-reported outcome (PRO) measures: ISPOR ePRO Good Research Practices Task Force report. Value Health. 2009;12(4):419-29.

31. Browne JP, O'Boyle CA, McGee HM, McDonald NJ, Joyce CR. Development of a direct weighting procedure for quality of life domains. Qual Life Res. 1997:6(4):301-9.

32. Evans MG. The problem of analyzing multiplicative composites: interactions revisited. Am Psychol. 1991;46(1):6-15.

33. Streiner DL, Norman GR, Cairney J. Health measurement scales: a practical guide to their development and use. Chapter 7: from items to scales. 5th ed. Oxford: Oxford University Press; 2015.

\section{Publisher's Note}

Springer Nature remains neutral with regard to jurisdictional claims in published maps and institutional affiliations. 